Prinzipien von derart mystischem Charakter eingeführt, daß hiedurch in einem unerfabrenen Leser der Anschein erweckt werden könnte, als ob die am Schlusse gegebenen praktischen Spielregeln in der Tat auf irgendwelche wissenschaftliche Prinzipien gegründet wären. In Wahrheit sind sie aber absolut wertlos und müssen genau ebenso wie das ganz gleiche planlose Spiel mit der Zeit zum Ruin des Spielers führen. Auf den Inhalt der Schrift, der vom Standpunkte exakter Wissenschaft als ejn ganz unsinniger bezeichnet werden muß, näher einzugehen, halten wir für zwecklos.

R. v. St.

Die Trigonometrie. Von Böttger und Hartenstein für den Unterricht an Realschulen bearbeitet, Dürrsche Buchharmllung, Leipzig, 1906. (60 १), $35 \mathrm{~S}$.

Ebene Trigonometrie im Umfang unseres Gymnasiallehrstoffes. Die änderung der Funktionen am ersten Quadranten des Einheitskreises bildet den Anfang.

F.

\title{
Urkunden zur Geschichte der Mathematik im Altertum.
} 1. Heft. Der Bericht des Simplicius über die Quadraturen des Antiphon und des Hippokrates. Griechisch und deutsch von Ferdinand Rudio. Mit 11 Figuren im Text. Leipzig, B. G. Teubner, $1907,8^{\circ} \mathrm{X}$. + 184 S. - Ladenpreis 4 M. 80 Pf.

Simplicius lebte in der ersten Hälfte des 6. Jahrhunderts nach Chr.

Sein Verdienst ist es, dorch seine Kommentare zu älteren philosophischen und mathematischen Werken uns wesentliche Bruchstücke dieser letzteren selbst überliefert zu haben. Dieses Verdienst fand in den Kreisen der Historiker der Philosophie seit langem die verdiente Anerkennung, während die Mathematiker von der geistigen Bedeutung des Simplicins meist eine recht ungünstige Meinung hatten und ihn für eine ungeschickten, wenn nicht geradezl unfähigen Menschen hielten. Der Grund dieser nunmehr als völlig irrig erkannten Geringschätzung ist in erster Linie darin zu sehen, daß das mathematische Publikum den Bericht des Simplicius bloß ans dem im Jahre 1870 erschienenen Werke von Bretschneider: „Die Geometrie und die Geometer vor Euklides“ kannte und diese Ausgabe, wie Radio schon im Jahre 1902 in einer in der „Bibliotheca nuathematica“" veröffentlichen Abhandlung nachwies, sowohl was den mitgeteilten Text als auch was die Übersetzung betrifft, als völlig ungenügend bezeichnet werden muß.

Den äußeren Anlaß, seinen Bericht zu schreiben, fand Simplicius in einer Bemerkung des Aristoteles, in der erklärt wird, daß man nicht alle falschen Sätze zu widerlegen brauche, sondern nur diejenigen, die nicht schon gegen die Prinzipien verstoßen. So sei es z. B. „Sache des Geometers, die Quadraturen vermittels der Segmente zu widerlegen, die des Antiphon aber za widerlegen sei nicht Sache des Geometers“. Da es nun nicht ganz deutlich war, welche der verschiedenen Methoden durch die Bezeichnung "Quadratur mittels der Segmente" gemeint sein sollte, so ging Simplicius die einzelnen Methoden durch und hat uns dadurch Arbeiten von großem wissensehaftlichen Werte überliefert, die ohne seinen Kommentar wahrscheinlich verloren gegangen wären, so namentlich die Untersuchungen von Hippokrates.

Die Ausgabe enthält jeweils auf der linken Seite den griechischen Originaltext, rechts die möglichst wörtliche Übersetzung ins Dentsche. Als 
Anhang sind noch einige ergänzende Urkunden and eine historische Übersicht der Kreisquadratur bei den Ägyptern und bei den Griechen bis Euklid, schließlich ein Wörter- and Namensverzeichnis beigefügt.

R. v. St.

I trattati delle sezioni coniche da Apollonio a Simson par F. Amodeo. Napoli, tipografia F. Giannini, 1906, 80. $51 \mathrm{~S}$.

Eine Antrittsvorlesung, die der Verfasser bei der Übernahme der Professur für Geschichte der Mathematik an der Universität Neapel hielt. Der Vortragende gibt zunächst einen Überblick über die sukzessive Einführung von Vorlesungen aus diesem Fache an den Universitäten der verschiedenen Staaten und entwickelt im Anschlusse daran seine Ideen über die Aufgaben und Probleme der Geschichtė der Mathematik. Dann geht der Verfasser auf das eigentliche Thema seines Vortrages über und gibt in einer durch die vollendete stilistische Durcharbeitung höchst anziehenden Form eine übersichtliche Darstellung derjenigen mathematischen Werke, durch die die wesentlichsten Fortschritte in der Theorie der Kegelschnitte gekennzeichnet sind, wobei er namentlich bei Apollonius, Kepler, Mydorge, Desargues, Pascal, De la Hire und Simson länger verweilt. Die vorliegende Antrittsvorlesung stellt einen Auszug ans einer gröBeren historischmathematischen Arbeit vor, die der Verfasser zu veröffentlichen beabsichtigt. R. v. St.

Abhandlungen zur Geschichte der mathematischen Wissenschaften mit Einschluß ihrer Anwendungen, begrïndet von Moritz Cantor. XXII. Heft. Briefwechsel zwischen C. G. J. Jacobi und M. H. Jacobi, herausgegeben von W. Ahrens. Mit zwei Bildnissen. Leipzig; B. G. Teubner, 1907, $8^{0}-\mathrm{XX}+282$ S. Ladenpreis 6 M. 90 Pf.

Es ist der Briefwechsel zwischen zwei sehr berühmten Brüdern, den W. Ahrens hiemit in möglichster Vollständigkeit veröffentlicht. Vor dem Titelblatte des Bandes finden wir die sehön ausgeführten Porträts der beiden Brüder. Der geniale Mathematiker Carl Gustav Jacob Jacobi, geb. 1804, gest. 1851, lebte von 1827 bis 1842 in Königsberg als Professor an der Universität, von da an als Akademiker und königlicher Pensionär in Berlin. Sein Bruder Moritz Hermann Jacobi, geb. 1801, gest. 1874, war anfangs preuBischer Baubeamter in Königsberg, dann von 1835 an Professor der Zivilbaukunst an der Universität Dorpat; im Jahre 1837 wurde er nach Petersburg berufen, wo er im Jahre 1842 außerordentliches und 1847 ordentliches Mitglied der Akademie wurde. Wenn auch seine Bedeutung nicht an die C. G. J. Jacobis heranreicht, so erlangte er doch, namentlich als Erfinder der Galvanoplastik, gleichfalls eine große Berühmtneit. Der vorliegende Band enthält 76 Briefe, 48 von C. G. J. Jacobi und 28 von M. H. Jacobi; sie fallen in die Jahre 1822 bis 1851. Wir erhalten aus denselben vielfach ganz intime Einblicke in das Gelehrtenleben der damaligen Zeit und lernen $\mathrm{u}$. a in allen Details Vorgänge in den gelehrten Akademien, denen die beiden Brüder angehörten, kennen. In den Jahren 1847 und 1848 spielen überdies die politischen Ereignisse, an denen C. G. J. Jacobi sich lebhaft beteiligte, eine bedeutende Rolle. Der Heransgeber hat keine Mühe gescheut, durch zahlreiche Anmerkungen, die er jedem einzelnen Briefe folgen läBt, die vielen persönlichen und literarischen Anspielungen, die in den Briefen vorkommen, dem Leser verständlich zu 